\title{
Oxidative degradation of p-chlorophenol by the persulfate-doped Fe-Mn bimetallic hydroxide, the parametrical significance, and systematical optimization
}

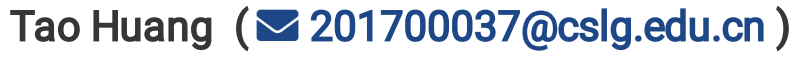

Changshu Institute of Technology https://orcid.org/0000-0002-8367-1218

Jing Du

Changshu Institute of Technology

Danni Yu

Changshu Institute of Technology

Shihan Deng

Changshu Institute of Technology

Shuwen Zhang

University of South China

Longfei Liu

Changshu Institute of Technology

\section{Research Article}

Keywords: Persulfate-doped Fe-Mn hydroxide, P-chlorophenol degradation, Optimization, Degradation kinetics, Systematical optimization

Posted Date: April 22nd, 2021

DOI: https://doi.org/10.21203/rs.3.rs-306271/v1

License: (9) This work is licensed under a Creative Commons Attribution 4.0 International License. Read Full License

Version of Record: A version of this preprint was published at Environmental Science and Pollution Research on August 5th, 2021. See the published version at https://doi.org/10.1007/s11356-021-157933. 
1 Oxidative degradation of p-chlorophenol by the persulfate-doped Fe-Mn bimetallic hydroxide, the parametrical significance, and systematical optimization Tao Huang ${ }^{\text {a }}{ }^{*}$, Jing Du ${ }^{\text {a }}$, Danni Yu ${ }^{a}$, Shihan Deng ${ }^{\text {a }}$, Shuwen Zhang ${ }^{\text {b }}$, Longfei Liu ${ }^{\text {a }}$ ${ }^{a}$ School of Materials Engineering, Changshu Institute of Technology, 215500, China *Email address: 201700037@.cslg.edu.cn (Tao Huang); 286809786@qq.com (Jing Du) 


\section{Abstract}

Aqueous Fe(II)-catalyzed activation commonly deteriorates the oxidation performance of persulfate (PS) hydroxide) was synthesized to construct a heterogeneously catalytic system to solve the issue brought by homogeneity. The molar ratio of $\mathrm{Fe}(\mathrm{II})$ to $\mathrm{Mn}$ (II) and the mass ratio of PS to Fe-Mn hydroxide both had a significant impact on the catalytic degradation of $\mathrm{p}$-CP. Reaction temperatures engaged in the most essential role in influencing the degradation and removal of p-chlorophenol (p-CP). The optimal combination of factors for the preparation of PS-hydroxide and the treatment of $\mathrm{p}$-CP was finally determined by significance analysis. The degradation process was appropriately fitted by the pseudofirst-order kinetic model. the benzene ring in p-CP was broken by PS-hydroxide during the adsorption. The surface modification of PS-hydroxide caused by the valence transition of Mn was beneficial to the adsorption and catalytic degradation of p-CP.

Capsule

A persulfate-doped layered bimetallic hydroxide was synthesized to construct a heterogeneously catalytic system to solve the drawback of homogeneity for the effective treatment of p-chlorophenol.

\section{Keywords}




\section{Introduction}

Chlorinated phenols (Cl-phenols) are extensively used in some chemical and pharmaceutical-related industries, which are commonly considered as persistent organic pollutants (POPs) in the treatment of wastewater(Mikkonen et al. 2018, Tubic et al. 2019). As a vital member of Cl-phenols, p-chlorophenol (p-CP) can cause severe ecological harm to the surrounding environment and humans, such as carcinogenicity, acute toxicity, and recalcitrance(Pirzada et al. 2019, Sharma \&Khare 2018). Therefore, it is essential to explore some effective technologies for the rapid removal of $\mathrm{p}$-CP to avoid further contamination. Until now, some techniques have been proposed and applied on the remediation of wastewater containing $\mathrm{p}-\mathrm{CP}$, such as extraction, adsorption, membrane separation, electrochemical oxidation, zero-valent iron (ZVI) reduction, and biological degradation(Bao et al. 2019, Jin \&Dong 2019, Kuntail et al. 2019, Wei et al. 2019). However, some shortcomings such as the large dosage of chemicals, low removal efficiency, a long-lasting disposal cycle, and the secondary pollution drawn by the secondary organic species have perplexed and dwarfed the wide application of these traditional technologies(Ababneh et al. 2019, Kim et al. 2019). In recent years, the advanced-oxidation methods (AOP) have attracted more attention particularly in treating non-biodegradable POPs in the wastewater due to their operational convenience and effectiveness(El Fakir et al. 2019, Lv et al. 2020, Xiao et al. 2020a). Among the oxidants, the utilization of hydroxyl radical $(\bullet \mathrm{OH})$ for the oxidation degradation of some organic compounds is most mentioned in various treatment processes(Laysandra et al. 2019, Shokoohi et al. 2019, Xiao et al. 2020b). $\bullet \mathrm{OH}$ has a higher potential compared with other common oxidants. Furthermore, $\bullet \mathrm{OH}$ can be easily produced by 
several technologies such as Fenton, ozonation, photocatalysis, electrocatalysis, and photoelectrocatalysis(Das et al. 2019, Li et al. 2020, Wang et al. 2020, Zhou et al. 2019). Nevertheless, the redox potential of $\bullet \mathrm{OH}$ is changeable in different $\mathrm{pH}$ environments (e.g., $+2.7 \mathrm{~V}$ in acidity and $+1.8 \mathrm{~V}$ in neutrality), which explicitly weakened its performance to some extent. Comparably, due to the distinctive solubility and operativity in the wide $\mathrm{pH}$ range, the sulfate radical $\left(\mathrm{SO}_{4}^{--}, \mathrm{E}_{0}=2.60 \mathrm{~V}\right)$-based heterogeneous oxidation has attracted more favor in degrading POPs in different $\mathrm{pH}$ environments (Guerra-Rodriguez et al. 2018, Huang \&Zhang 2019, Liu et al. 2018).

$\mathrm{SO}_{4}{ }^{--}$can be generated by activating persulfate (PS) under ambient or heating conditions(Crincoli et al. 2020, Xu et al. 2020). The activation of PS is commonly conducted by some methods, such as heating, microwave, transition-metal catalysis, UV radiation, and electrolysis, etc. Generally, among these pathways, the transition-metal catalysis with the participation of aqueous $\mathrm{Fe}^{2+}$ is preferably adopted for the activation of PS in the application due to its lower temperature request and running cost(Dong et al. 2019, Jin \&Dong 2019). However, the relatively hard control of activation rate and reaction disturbance brought by excess aqueous $\mathrm{Fe}(\mathrm{II})$ cations would severely deteriorate the oxidation performance of $\mathrm{SO}_{4}^{*}$ on treating organic pollutants. An excessive loss rate of $\mathrm{Fe}(\mathrm{II})$ would further hamper the employment of the homogeneous activator when the $\mathrm{pH}$ in the environment is higher than 5 . Recently, some heterogeneous activators such as iron oxide, iron-based minerals, nanoscale zero-valent iron, etc. have been used to substitute aqueous $\mathrm{Fe}^{2+}$ to potentially enhance the PS activation(Deng et al. 2019, Jin \&Dong 2019, Jin et al. 2019, Park et al. 2019). However, the pre-judgment effect of the control in releasing Fe(II) ions is still poor. $\mathrm{Mn}(\mathrm{II})$ cations besides $\mathrm{Fe}(\mathrm{II})$ can also activate PS to produce $\mathrm{SO}_{4}{ }^{-{ }^{-}}$at a slower rate by transforming its bivalence to a higher valence state(Huang \&Zhang 2019, Shah et al. 2019). Moreover, 
the Mn species with a higher valence state is anticipated to vastly adsorb the excessive $\mathrm{Fe}(\mathrm{II})$ and $\mathrm{Fe}(\mathrm{III})$ to maintain the continuous degradation process(Eyvazi et al. 2019, Lin et al. 2019).

To solve the abovementioned issues, the layered bimetallic Fe-Mn hydroxide was synthesized as the heterogeneous activator of PS in this study. PS was doped to the bimetallic hydroxide using a mechanochemical method to increase the activation activities. To directly characterize the influence of the preparation parameters and the aqueous conditions on the oxidation performance of the PS-doped bimetallic hydroxide (PS-hydroxide), two orthogonal designs with seven factors in total were arranged and quantitatively analyzed based on the removal amounts of $\mathrm{p}$-CP. The synthesis and treatment processes were optimized by the significance analysis and the marginal results. To specify the degradation mechanisms of p-CP by PS-hydroxide, FTIR, SEM, and EDS (i.e., Energy dispersive x-ray spectroscopy) were employed in combination for the characterization analysis of the solid and aqueous samples obtained from different conditions. This study solves the performance issue of PS drawn by the aqueous $\mathrm{Fe}(\mathrm{II})$-activated drawback for the degradation of $\mathrm{p}-\mathrm{CP}$ and clarifies the role of $\mathrm{Mn}(\mathrm{II})$ in the iron-dominated catalysis process.

\section{Methods and materials}

\subsection{Chemicals}

All chemical reagents including sodium hydroxide $(\mathrm{NaOH})$, ferrous sulfate heptahydrate $\left(\mathrm{FeSO}_{4} \cdot 7 \mathrm{H}_{2} \mathrm{O}\right)$, manganese chloride tetrahydrate $\left(\mathrm{MnCl}_{2} \cdot 4 \mathrm{H}_{2} \mathrm{O}\right)$, potassium persulfate $\left(\mathrm{K}_{2} \mathrm{~S}_{2} \mathrm{O}_{8}, \mathrm{PS}\right)$, and p-chlorophenol (p-CP) were of analytical grade, purchased online from Aladdin Reagent Company (China), and stored in a vacuum environment. Deionized (DI) water used for the experiments was generated by a water 
purification device (Super 10FV, HHITECH, China), boiled, and purged with nitrogen gas (95\% $\mathrm{N}_{2}$ and $5 \% \mathrm{H}_{2}$ ) to remove the dissolved oxygen gas.

\subsection{Preparation of the PS-doped Fe(II)-Mn(II) hydroxide}

All the glass containers and gadgets have been rinsed three times using DI water and dried before any use. The whole preparation process was conducted in a vacuum glove box to avoid the oxidation of Fe(II) and $\mathrm{Mn}(\mathrm{II})$ by $\mathrm{O}_{2}$. The Fe-Mn bimetallic hydroxide was synthesized by the co-precipitation of the dissolved $\mathrm{Fe}(\mathrm{II})$ and $\mathrm{Mn}$ (II) species before the synthesis of the PS-doped Fe-Mn oxidative inducer. $\mathrm{FeSO}_{4} \cdot 7 \mathrm{H}_{2} \mathrm{O}$ and $\mathrm{MnCl}_{2} \cdot 4 \mathrm{H}_{2} \mathrm{O}$ were weighted at the molar ratios of $\mathrm{Fe}(\mathrm{II})$ to $\mathrm{Mn}(\mathrm{II})$ of 1,3 , and 5 , respectively, and both dissolved in DI water. $\mathrm{NaOH}$ was weighted at some molar ratios of $\mathrm{OH}^{-}$and $\mathrm{Fe}(\mathrm{II})$ and dissolved to obtain an alkaline solution. The dark-green flocculated precipitation was immediately generated once the alkaline solution was poured into the water containing $\mathrm{Fe}(\mathrm{II})$ and $\mathrm{Mn}$ (II) cations. Afterward, the mixture was mechanically stirred to make full precipitation, sequentially centrifuged using a centrifuge (KH19A, KAIDA, China) at $8000 \mathrm{rpm}$, and dried at $75^{\circ} \mathrm{C}$ by a vacuum drying oven (ZK-50A, Biao Cheng Share Laboratory Co. Ltd, China). Finally, the PS-doped Fe-Mn hydroxide (PShydroxide) was prepared by mixing Fe-Mn hydroxide with PS at some mass ratios (i.e., the mass ratios of PS to Fe-Mn hydroxide of $1-5$ ) and mechanically milling at 120 rpm over 2 hours (h). The PShydroxide samples were stored in the glovebox for further employment.

\subsection{Experimental procedure}

A certain amount of oxidative reducer (the PS-hydroxide) was weighted and added to the p-CP stock solution in the Erlenmeyer flask $(250 \mathrm{~mL})$. A water-bath shaker (COS-100B, Shanghai BILON 
INSTRUMENTS Co. LTD, China) was employed to control the shaking time (6 h) and the temperature of the solution. The influence of the preparation parameters (including the molar ratio of $\mathrm{Fe}(\mathrm{II})$ to $\mathrm{Mn}$ (II) (Fe/Mn ratio), the mass ratio of PS to Fe-Mn hydroxide (PS/Fe-Mn ratio), and the molar ratio of $\mathrm{OH}^{-}$to $\mathrm{Fe}(\mathrm{II})\left(\mathrm{OH}^{-} / \mathrm{Fe}\right.$ ratio $\left.)\right)$ and the aqueous conditions (including the initial $\mathrm{pH}$, concentration, PS-hydroxide, and temperature) on the degradation and removal of $\mathrm{p}-\mathrm{CP}$ were comprehensively analyzed in batch experiments. Two orthogonal designs with different factors and levels were constructed, respectively, to optimize the synthesis of the PS-hydroxide and effectively arrange the degradation experiments affected by the changes in the preparation parameters and the aqueous conditions. Variance analysis (ANOVA) was used to obtain marginal means and quantitively evaluate the significance of each factor. The factors and levels with specific labels are correspondingly listed in Table S1 and Table S2 in the supporting information (SI). The orthogonal designs with the p-CP removal results are shown in Table 1 and Table model was adopted to describe the removal process following Eq. (1), where $c_{0}$ and $c_{t}$ are the concentrations of $\mathrm{p}-\mathrm{CP}$ in solutions at the initial and $\mathrm{t}$ times, respectively, $k$ is the constant reaction rate bounded to the physicochemical characteristics of PS-hydroxide $\left(\mathrm{h}^{-1}\right)$. $\ln \frac{c_{t}}{c_{0}}=-k t$

Liquid samples taken off at a specific time were centrifuged at $5000 \mathrm{rpm}$ and filtered through a membrane 
141 deviations. The samples of the P-CP solution and the PS-hydroxide before and after the degradation

142 process were characterized by a Fourier transform infrared spectrometer (FTIR, Thermo Electron Co.-

143380 FTIR, USA) over a range of $400-4000 \mathrm{~cm}^{-1}$ at every step of $4 \mathrm{~cm}^{-1}$ and a scanning electron

144 microscope (SEM) equipped with energy-dispersive X-ray spectroscopy (EDS, Carl Zeiss AG,

145 Germany).

\section{Results and discussion}

3.1 Characterization of Fe-Mn hydroxide and PS-hydroxide

FTIR spectra of Fe-Mn hydroxide affected by Fe/Mn ratio and the PS-hydroxide influenced by both

$\mathrm{Fe} / \mathrm{Mn}$ ratio and $\mathrm{PS} / \mathrm{Fe}-\mathrm{Mn}$ ratio are shown in Fig. 1. The morphologies and EDS distribution of samples are correspondingly displayed in Fig. 2 and Fig. S1 in the SI, respectively. The peaks at the wavenumbers $\left(\mathrm{cm}^{-1}\right)$ of $3420,1626,1384,1121,983,612$, and 465 were matched, respectively, at the Fe/Mn ratio of 1 for the Fe-Mn hydroxide (Fig. 1 a). However, the peak at $983 \mathrm{~cm}^{-1}$ disappeared in terms of the spectra at the Fe/Mn ratio of 3 and 5. Moreover, the peak at $465 \mathrm{~cm}^{-1}$ was shifted to $494 \mathrm{~cm}^{-1}$ for the Fe/Mn ratio of 3 and a brand peak at $571 \mathrm{~cm}^{-1}$ was newly formed for the $\mathrm{Fe} / \mathrm{Mn}$ ratio of 5 . The partial oxidation of $\mathrm{Fe}(\mathrm{II})$ and the substitution of Fe into the Mn-related hydroxide potentially analyzed the changes in the FTIR spectra of Fe-Mn hydroxide with the increase of Fe/Mn ratios(Chen et al. 2019, Lin et al. 2019). Comparably, more peaks were observed in the spectra of PS-hydroxide although the changes in the Fe/Mn ratios had an unobvious effect on the change of the spectra pattern (Fig. $1 \mathrm{~b}$ ). The results confirmed that the PS had been mechanochemically doped into the Fe-Mn hydroxide, which covered the change of spectra caused by Fe/Mn ratios(Cao et al. 2017). Furthermore, the peaks within the bands of $592-690 \mathrm{~cm}^{-1}$ and $1060-1274 \mathrm{~cm}^{-1}$ gradually disappeared with the increase of the PS/Fe-Mn ratio (Fig. 
$1 \mathrm{c})$, which reconfirmed the PS loading on the Fe-Mn hydroxide. The layered precipitation was observed in the SEM image of Fe-Mn hydroxide (Fig. 2 a). The morphologies of the Fe-Mn hydroxide confirmed that the increase of $\mathrm{Fe}$ in the hydroxide facilitated the formation of the layered bimetallic hydroxide (Fig. 2 a). The Fe-Mn hydroxide was tightly wrapped by the amorphous (also flocculated) PS in the PShydroxide (Fig. 2 b). The flocculated morphology of PS indicated the partial catalysis of PS to sulfate radicals, which further induced the oxidation of $\mathrm{Fe}(\mathrm{II})$ and $\mathrm{Mn}$ (II) and the precipitation of $\mathrm{Fe}$ and $\mathrm{Mn}$ cations with $\mathrm{OH}^{-}$. The flocculation would be beneficial to the capture of $\mathrm{p}-\mathrm{CP}$ in further degradation experiments. The intercalation of the layered hydroxide into PS was observed for all three PS/Fe-Mn ratios. The morphological characteristics of PS-hydroxide echoed with the analysis of FTIR spectra. The mass contents of Mn and Fe were 16.44 and 22.94, 10.94 and 34.95, and 4.74 and 24.79, respectively, which were roughly close to the corresponding Fe/Mn ratios (i.e., 1, 2, and 3) (Fig. S1, SI). Generally, the mass contents of $\mathrm{Fe}, \mathrm{Mn}$, and $\mathrm{S}$ semi-quantitatively reflected the reliability of $\mathrm{Fe} / \mathrm{Mn}$ ratios and $\mathrm{PS} / \mathrm{Fe}-$ Mn ratios.

The main effect of the preparation parameters on $\mathrm{p}-\mathrm{CP}$ removal (default $\alpha=0.05$ ) is shown in Table S3 in the SI. The quantitative results analyzed by ANONA were acceptable due to the $R^{2}$ value (R: regression coefficient) that was close to 1 . The significance coefficients (Sig.) were $0.040,0.038$, and 0.318 for factor $\mathrm{A}$ (i.e., $\mathrm{Fe} / \mathrm{Mn}$ ratio), B (i.e., $\mathrm{PS} / \mathrm{Fe}-\mathrm{Mn}$ ratio), and $\mathrm{C}$ (i.e., $\mathrm{OH}^{-} / \mathrm{Fe}$ ratio), respectively. The preparation parameters including A and B factors both had a significant effect on the removal of $\mathrm{p}-\mathrm{CP}$ using PS-hydroxide. Contrarily, the influence of the $\mathrm{OH}^{-} / \mathrm{Fe}$ ratio on the removal of $\mathrm{p}-\mathrm{CP}$ was unobvious. 
$\mathrm{A}>$ Factor $\mathrm{C}$. The parameter of $\mathrm{PS} / \mathrm{Fe}-\mathrm{Mn}$ ratio played a most important role among the factors in strengthening the performance of PS-hydroxide towards p-CP removal. The different morphologies of PS-hydroxide (Fig. 2 a and b) caused by the Fe/Mn ratio and PS/Fe-Mn ratio explicitly impacted the reactions between PS-hydroxide and p-CP to some extent. The main effect of the aqueous conditions on p-CP removal (default $\alpha=0.05$ ) is shown in Table S4 in the SI. The $R^{2}$ value of ANONA analysis was 0.822, indicating that the quantitative results listed in Table S4 were moderately acceptable. The Sig. values were $0.457,0.364,0.799$, and 0.012 for the factor $\mathrm{D}$ (i.e., Initial $\mathrm{pH}$ ), E (i.e., P-CP concentration), F (i.e., PS-hydroxide dosage), and G (i.e., Temperature), respectively. Only Sig. value of factor F among the aqueous conditions was lower than the default $\alpha$ of 0.05 , meaning only the temperature of the solution significantly affected the removal of p-CP achieved by PS-hydroxide. The impact of the other three factors on the removal results was insignificant while nonnegligible. Similarly, the significant order of the aqueous conditions followed the descending rank as Factor $\mathrm{G}>$ Factor $\mathrm{E}>$ Factor $\mathrm{D}>$ Factor F. Obviously, the variable of temperature engaged in the most essential role in alternating the degradation process of p-CP(Chao et al. 2018).

Marginal means of the preparation parameters and aqueous conditions in the orthogonal design (default $\alpha=0.05)$ are shown in Fig. 3. Although, the maximum removal amounts (mM) of 5.37 and 8.12 were obtained in the parametrical combination of $\mathrm{A}_{2} \mathrm{~B}_{1} \mathrm{C}_{3}$ (i.e., $\mathrm{Fe} / \mathrm{Mn}$ ratio of $3, \mathrm{PS} / \mathrm{Fe}-\mathrm{Mn}$ ratio of 1 , and $\mathrm{OH}^{-}$ /Fe ratio of 6) and $\mathrm{D}_{5} \mathrm{E}_{5} \mathrm{~F}_{3} \mathrm{G}_{5}$ (i.e., Initial $\mathrm{pH}$ of 11 , the $\mathrm{p}-\mathrm{CP}$ concentration of $10 \mathrm{mM}$, Dosage of $1.5 \mathrm{~g} / \mathrm{L}$, and Temperature of $70^{\circ} \mathrm{C}$ ) in the two orthogonal designs, respectively (Table 1 and Table 2). The results conflicted with the significance-analyzed ones (Table S3 and Table S4, SI), which cannot be used to 
represent the data in the full experiments with $3^{3}$ plus $4^{5}$ runs. The marginal means of factors combining with the significance analysis should be employed for the determination of systematical optimization. As seen in Fig. 3, for the factor of Fe/Mn ratio, the level (i.e., 1, 3, and 5) of $3\left(\mathrm{~A}_{2}\right)$ was most conducive to the removal peak of p-CP. Similarly, the factors with the specific levels including $B_{1}, C_{1}, D_{5}, E_{4}, F_{3}$, and Gs were assured as the most preferred values for the optimal removal means. Furthermore, the effect significances of all factors were ranked in the whole following $\mathrm{G}>\mathrm{B}>\mathrm{A}>\mathrm{C}>\mathrm{E}>\mathrm{D}>\mathrm{F}$ under the significance results (Table S3 and Table S4, SI). It was clear that Dosage, Initial $\mathrm{pH}$, and $\mathrm{p}-\mathrm{CP}$ concentration were the three least significant factors. Therefore, the medium levels were preferred to maintain cost-effectiveness to some extent. The high temperature in the solution (e.g., 60 and $70{ }^{\circ} \mathrm{C}$ ), the low $\mathrm{PS} / \mathrm{Fe}-\mathrm{Mn}$ ratios (e.g., 1), and the medium $\mathrm{Fe} / \mathrm{Mn}$ ratio (e.g., 3) were selected in favor of the significant importance of the three factors. As a result of the analysis, the optimal combination of factors for the preparation of PS-hydroxide and the treatment of p-CP was finalized for $\mathrm{A}_{2} \mathrm{~B}_{1} \mathrm{C}_{2} \mathrm{D}_{3} \mathrm{E}_{3} \mathrm{~F}_{3} \mathrm{G}$.

\subsection{Kinetics description for $\mathrm{p}-\mathrm{CP}$ removal}

Removal amounts of $\mathrm{p}-\mathrm{CP}$ affected by the preparation parameters and the aqueous conditions over time

(360 min) are shown in Fig. 4. For the factor of Fe/Mn ratio, an increasing curve with a slower rate before

a fast one over the whole running time was observed at all three levels. The PS/Fe-Mn ratio performed a similar trend in comparison with the $\mathrm{Fe} / \mathrm{Mn}$ ratio. However, the $\mathrm{OH}^{-} / \mathrm{Fe}$ ratio influenced the removal curves differently with a nearly constant rate obtained in the middle and later stages of experiments. However, the removal process of $\mathrm{p}-\mathrm{CP}$ was intensely changed by the change of the initial $\mathrm{pH}$ in the middle-later phase (i.e., from 210 until to $360 \mathrm{~min}$ ). A high p-CP concentration (e.g., 6, 8, and $10 \mathrm{mM}$ ) enhanced the removal rate of p-CP for the PS-hydroxide generally despite its inconspicuous significance 
analysis (Table S4, SI). The increase in the dosage of PS-hydroxide based on the medium value (i.e., 2.0 and $2.5 \mathrm{~g} / \mathrm{L}$ ) had not supported the continuing elevation of $\mathrm{p}-\mathrm{CP}$ removal although the reaction rate was strengthened in the initial and middle stage of the experiment (i.e., before $240 \mathrm{~min}$ ). Notably, the temperature factor generated the largest removal difference in the level range among the seven factors. The significance of temperature was visually confirmed by the different removal curves over time. The linear fit of the pseudo-first-order kinetic model affected by the preparation parameters and aqueous conditions is shown in Fig. 5. The parametrical statistics for the kinetics fitting are correspondingly listed in Table S5 in the SI. As seen, most of the adjusted $R^{2}$ values (i.e., Adj. $R^{2}$ ) were larger than the default value of 0.95 . Overall, the change of $\ln \left(\mathrm{c}_{\mathrm{t}} / \mathrm{c}_{0}\right)$ vs time can be appropriately fitted by the pseudo-first-order kinetic model. The suitableness of the model directly clarified that the removal of p-CP mostly endured a heterogeneous mechanism containing both physical and chemical pathways(Bao et al. 2019, Pirzada et al. 2019, Wan \&Li 2017). Most p-CP was adsorbed onto the surface of hydroxide before the subsequent immediate oxidation by $\mathrm{SO}_{4}^{--}$with only a minor moiety was degraded through a homogeneous process(Brienza \&Katsoyiannis 2017).

FTIR spectra of $\mathrm{p}-\mathrm{CP}$ before and after the degradation process and the PS-hydroxide after the degradation process are shown in Fig. 6. As seen, fewer peaks were matched in the spectra of the degraded p-CP compared with the raw sample (Fig. 6 a). The increase in the concentration of p-CP would increase the number of peaks. To analyze the changes in the molecular structure of $\mathrm{p}-\mathrm{CP}$ before and after experiments, the FTIR spectrum of benzene was analyzed and shown in Fig. S2 in the SI. The standard peaks of 
the peaks at 3447 and $1384 \mathrm{~cm}^{-1}$, the spectra of the degraded p-CP were different from benzene. The benzene ring in $\mathrm{p}-\mathrm{CP}$ had been broken by PS-hydroxide during the test. The peak at $3447 \mathrm{~cm}^{-1}$ is commonly associated with the $\mathrm{O}-\mathrm{H}$ band in the water molecular. The peaks at 1634,1429 , and $1384 \mathrm{~cm}^{-}$ ${ }^{1}$ represent $\mathrm{C}=\mathrm{O}, \mathrm{C}-\mathrm{C}$, and $\mathrm{C}=\mathrm{C}$ bonds, respectively(He et al. 2015, Huang et al. 2017, Taherian et al. 2013). The results indicated that $\mathrm{p}-\mathrm{CP}$ had experienced an incomplete mineralization process potentially with some by-products being produced. Similarly, fewer peaks were matched in the spectra of PShydroxide after the degradation (Fig. 6 b) in comparison with the raw counterpart (Fig. 1 b, c). The morphology of the PS-hydroxide after the degradation process is shown in Fig. 7. Some different morphological characteristics were observed for the used PS-hydroxide sample. A complicated physicochemical mechanism foreseeably participated in the degradation process. PS is activated into $\mathrm{SO}_{4}^{--}$radical mainly by heat, UV (also light-driven) radiation, transition metal ions, and electrolysis(Crincoli et al. 2020, Forouzesh et al. 2019). Generally, the reaction mechanism can be substantially surmised based on the above analysis and results. PS doped into the Fe-Mn hydroxide was dissolved into the solid-liquid interface once PS-hydroxide was added to the solution (Fig. 7), which thereupon was activated by ferrous ions to $\mathrm{SO}_{4}^{--}$(Eq. 2) that dominantly participated in the dechlorination, loop disintegration, carbon-chain breaking, and mineralization processes (Table S3, Fig. 4, and Fig. 6)(Nguyen \&Juang 2015, Taherian et al. 2013). Divalent Mn was oxidized to tetravalent species accompanying the activation of PS involving Fe(II) ions (Eq. 4, Fig. 5 and Table S5, SI), which induced more pores, cracks, and flocculation on PS-hydroxide (Fig. 7). Considering the removal of p-CP by PShydroxide mainly was a heterogeneous process (Fig. 5 and Table S5), the surface modification of the PShydroxide caused by the valence transition of Mn was beneficial to the adsorption of $\mathrm{p}-\mathrm{CP}$ and subsequent removal or degradation. However, too high or too low $\mathrm{Fe} / \mathrm{Mn}$ ratios (i.e., $\mathrm{A}_{1}$ and $\mathrm{A}_{3}$ ) were both 


\section{Conclusion}

PS was mechanochemically doped into the layered Fe-Mn hydroxide to solve the homogenous issue for the degradation of $\mathrm{p}-\mathrm{CP}$, which caused the amorphous and flocculation in the PS-hydroxide. The impact of the $\mathrm{Fe} / \mathrm{Mn}$ ratio, the $\mathrm{PS} / \mathrm{Fe}-\mathrm{Mn}$ ratio, and the temperature on the removal of $\mathrm{p}-\mathrm{CP}$ was significant among all factors. The quantitative influence of factors on the removal of $\mathrm{p}-\mathrm{CP}$ ranked as $\mathrm{G}>\mathrm{B}>\mathrm{A}>$ $\mathrm{C}>\mathrm{E}>\mathrm{D}>\mathrm{F}$. The maximum removal amounts $(\mathrm{mM})$ of 5.37 and 8.12 were obtained in the parametrical combination of $\mathrm{A}_{2} \mathrm{~B}_{1} \mathrm{C}_{3}$ and $\mathrm{D}_{5} \mathrm{E}_{5} \mathrm{~F}_{3} \mathrm{G}_{5}$ in the two orthogonal designs, respectively. The optimal combination of $\mathrm{A}_{2} \mathrm{~B}_{1} \mathrm{C}_{2} \mathrm{D}_{3} \mathrm{E}_{3} \mathrm{~F}_{3} \mathrm{G}_{5}$ was determined for the preparation of PS-hydroxide and the treatment of p-CP based on the significance analysis and the marginal means of the experimental results. The linear fit of the pseudo-first-order kinetic model was confirmed for the preparation parameters and aqueous conditions, which demonstrated that most $\mathrm{p}-\mathrm{CP}$ was adsorbed onto the surface of hydroxide and then experienced the immediate oxidation by $\mathrm{SO}_{4}^{--}$. The spectra of the degraded $\mathrm{p}-\mathrm{CP}$ were different from the standard peaks of benzene. The benzene ring in p-CP had been broken by PS-hydroxide during the 


\section{Declarations}

299 Ethics approval and consent to participate-

$300 \quad$ Not applicable

301 Consent for publication

Not applicable

Availability of data and materials

The datasets generated and/or analyzed during the current study are available in the [NAME] repository,

Competing interests

The authors declare that they have no competing interests

308 Funding

309 The China Postdoctoral Science Foundation (2020M681774), the Natural Science Foundation of the Jiangsu Higher Education Institutions of China (20KJB490001), and Xuzhou Science and Technology Bureau (Nos. KC19228 and KC20055).

Authors' contributions conception and design. Material preparation, data collection, and analysis were performed by Tao commented on previous versions of the manuscript. Tao Huang, Jing Du, Longfei Liu, and Shuwen Zhang 
read and approved the final manuscript.

\section{References}

Ababneh AN, Abu-Dalo MA, Horn C, Hernandez MT (2019): Polarographic determination of benzotriazoles and their sorption behavior on granular activated carbon. Int J Environ Sci Te 16, 833-842

Bao T, Jin J, Damtie MM, Wu K, Yu ZM, Wang L, Chen J, Zhang Y, Frost RL (2019): Green synthesis and application of nanoscale zero-valent iron/rectorite composite material for P-chlorophenol degradation via heterogeneous Fenton reaction. J Saudi Chem Soc 23, 864-878

Brienza M, Katsoyiannis IA (2017): Sulfate Radical Technologies as Tertiary Treatment for the Removal of Emerging Contaminants from Wastewater. Sustainability-Basel 9

Cao D, Zeng HB, Yang B, Zhao X (2017): Mn assisted electrochemical generation of two-dimensional Fe-Mn layered double hydroxides for efficient $\mathrm{Sb}(\mathrm{V})$ removal. J Hazard Mater 336, 33-40

Chao HP, Wang YC, Tran HN (2018): Removal of hexavalent chromium from groundwater by Mg/Allayered double hydroxides using characteristics of in-situ synthesis. Environ Pollut 243, 620629

Chen G, Nengzi LC, Li B, Gao YJ, Zhu GX, Cheng XW (2019): Octadecylamine degradation through catalytic activation of peroxymonosulfate by Fe-Mn layered double hydroxide. Sci Total Environ 695

Crincoli KR, Green C, Huling SG (2020): Sulfate Radical Scavenging by Mineral Surfaces in PersulfateDriven Oxidation Systems: Reaction Rate Constants and Implications. Environ Sci Technol 54, 1955-1962 
Das KK, Patnaik S, Nanda B, Pradhan AC, Parida K (2019): ZnFe2O4-Decorated Mesoporous Al2O3 Modified MCM-41: A Solar-Light-Active Photocatalyst for the Effective Removal of Phenol and Cr (VI) from Water. Chemistryselect 4, 1806-1819

Deng JM, Dong HR, Li L, Wang YY, Ning Q, Wang B, Zeng GM (2019): Ca(OH)(2) coated nanoscale zero-valent iron as a persulfate activator for the degradation of sulfamethazine in aqueous solution. Sep Purif Technol 227

Dong HR, Hou KJ, Qiao WW, Cheng YJ, Zhang LH, Wang B, Li L, Wang YY, Ning Q, Zeng GM (2019): Insights into enhanced removal of TCE utilizing sulfide-modified nanoscale zero-valent iron activated persulfate. Chem Eng J 359, 1046-1055

El Fakir AA, Anfar Z, Benafqir M, Jada A, El Alem N (2019): Polyaniline coated hematite sand supported on graphene oxide (HS@PANI-GO) as a new magnetic material for advanced catalytic oxidation based on sulfate radicals: optimization using response surface methodology. J Chem Technol Biot 94, 2609-2620

Eyvazi B, Jamshidi-Zanjani A, Darban AK (2019): Immobilization of hexavalent chromium in contaminated soil using nano-magnetic MnFe2O4. J Hazard Mater 365, 813-819

Forouzesh M, Ebadi A, Aghaeinejad-Meybodi A, Khoshbouy R (2019): Transformation of persulfate to free sulfate radical over granular activated carbon: Effect of acidic oxygen functional groups. Chem Eng J 374, 965-974

Guerra-Rodriguez S, Rodriguez E, Singh DN, Rodriguez-Chueca J (2018): Assessment of Sulfate Radical-Based Advanced Oxidation Processes for Water and Wastewater Treatment: A Review. Water-Sui 10

He ZQ, Jiang LX, Wang D, Qiu JP, Chen JM, Song S (2015): Simultaneous Oxidation of p-Chlorophenol 
and Reduction of $\mathrm{Cr}(\mathrm{VI})$ on Fluorinated Anatase TiO2 Nanosheets with Dominant $\{001\}$ Facets under Visible Irradiation. Ind Eng Chem Res 54, 808-818

Huang JZ, Zhang HC (2019): Mn-based catalysts for sulfate radical-based advanced oxidation processes: A review. Environ Int 133

Huang ZJ, Wu PX, Gong BN, Zhang X, Liao ZC, Chiang PC, Hu XJ, Cui LH (2017): Immobilization of visible light-sensitive $(\mathrm{N}, \mathrm{Cu})$ co-doped $\mathrm{TiO} 2$ onto rectorite for photocatalytic degradation of $\mathrm{p}$ chlorophenol in aqueous solution. Appl Clay Sci 142, 128-135

Jin H, Dong J (2019): Enhanced performance of Ag3PO4/Fe3O4/GO bifunctional catalysts on pchlorophenol degradation in advanced catalytic oxidation systems. Colloid Surface A 581

Jin YY, Wang XN, Sun SP, Dong WB, Wu ZX, Bian GQ, Wu WD, Chen XD (2019): Hydroxyl and sulfate radicals formation in UVA/Fe-III-NTA/S2O82- system: Mechanism and effectiveness in carbamazepine degradation at initial neutral pH. Chem Eng J 368, 541-552

Kim HB, Kim JG, Kim SH, Kwon EE, Baek K (2019): Consecutive reduction of Cr(VI) by Fe(II) formed through photo-reaction of iron-dissolved organic matter originated from biochar. Environ Pollut $253,231-238$

Kuntail J, Jain YM, Shukla M, Sinha I (2019): Adsorption mechanism of phenol, p-chlorophenol, and pnitrophenol on magnetite surface: A molecular dynamics study. J Mol Liq 288

Laysandra L, Ondang IJ, Ju YH, Ariandini BH, Mariska A, Soetaredjo FE, Putro JN, Santoso SP, Darsono FL, Ismadji S (2019): Highly adsorptive chitosan/saponin-bentonite composite film for removal of methyl orange and Cr(VI). Environ Sci Pollut R 26, 5020-5037

Li HX, Miao XR, Zhang J, Du J, Xu SD, Tang JH, Zhang YY (2020): DFT studies on the reaction mechanism and kinetics of dibutyl phthalate initiated by hydroxyl and sulfate radicals: 
Lin LN, Song ZG, Huang YC, Khan ZH, Qiu WW (2019): Removal and Oxidation of Arsenic from Aqueous Solution by Biochar Impregnated with Fe-Mn Oxides. Water Air Soil Poll 230

Liu CG, Wu B, Chen X (2018): Sulfate radical-based oxidation for sludge treatment: A review. Chem Eng J 335, 865-875

Lv SW, Liu JM, Li CY, Zhao N, Wang ZH, Wang S (2020): Two novel MOFs@COFs hybrid-based photocatalytic platforms coupling with sulfate radical-involved advanced oxidation processes for enhanced degradation of bisphenol A. Chemosphere 243

Mikkonen A, Ylaranta K, Tiirola M, Dutra LAL, Salmi P, Romantschuk M, Copley S, Ikaheimo J, Sinkkonen A (2018): Successful aerobic bioremediation of groundwater contaminated with higher chlorinated phenols by indigenous degrader bacteria. Water Res 138, 118-128

Nguyen AT, Juang RS (2015): Photocatalytic degradation of p-chlorophenol by hybrid $\mathrm{H} 2 \mathrm{O} 2$ and $\mathrm{TiO} 2$ in aqueous suspensions under UV irradiation. J Environ Manage 147, 271-277

Park JH, Wang JJ, Tafti N, Delaune RD (2019): Removal of Eriochrome Black T by sulfate radical generated from Fe-impregnated biochar/persulfate in Fenton-like reaction. J Ind Eng Chem 71, 201-209

Pirzada BM, Pushpendra, Kunchala RK, Naidu BS (2019): Synthesis of LaFeO3/Ag2CO3 Nanocomposites for Photocatalytic Degradation of Rhodamine B and p-Chlorophenol under Natural Sunlight. Acs Omega 4, 2618-2629

Shah NS, Khan JA, Sayed M, Khan ZU, Ali HS, Murtaza B, Khan HM, Imran M, Muhammad N (2019): Hydroxyl and sulfate radical mediated degradation of ciprofloxacin using nano zerovalent manganese catalyzed S2O82-. Chem Eng J 356, 199-209 
Sharma S, Khare N (2018): Hierarchical Bi2S3 nanoflowers: A novel photocatalyst for enhanced photocatalytic degradation of binary mixture of Rhodamine B and Methylene blue dyes and degradation of mixture of p-nitrophenol and p-chlorophenol. Adv Powder Technol 29, 33363347

Shokoohi R, Bajalan S, Salari M, Shabanloo A (2019): Thermochemical degradation of furfural by sulfate radicals in aqueous solution: optimization and synergistic effect studies. Environ Sci Pollut R 26, 8914-8927

Taherian S, Entezari MH, Ghows N (2013): Sono-catalytic degradation and fast mineralization of pchlorophenol: $\mathrm{La} 0.7 \mathrm{Sr} 0.3 \mathrm{MnO} 3$ as a nano-magnetic green catalyst. Ultrason Sonochem 20, $1419-1427$

Tubic A, Loncarski M, Maletic S, Jazic JM, Watson M, Trickovic J, Agbaba J (2019): Significance of Chlorinated Phenols Adsorption on Plastics and Bioplastics during Water Treatment. Water-Sui 11

Wan YS, Li DW (2017): Removal of p-chlorophenol in wastewater through catalytic ozonation using MnOx/A12O3. Bulg Chem Commun 49, 200-204

Wang AQ, Chen Z, Zheng ZK, Xu H, Wang H, Hu K, Yan K (2020): Remarkably enhanced sulfate radical-based photo-Fenton-like degradation of levofloxacin using the reduced mesoporous MnO@MnOx microspheres. Chem Eng J 379

Wei X, Zeng LY, Miao J, Zhang RC, Zhang JJ, An S, Zhang J (2019): Electrocatalytic dechlorination of p-chlorophenol using a 3D cathode with magnetic separable Fe3O4@PPy@Pd catalyst. Int J Electrochem Sc 14, 11178-11188

Xiao RY, He L, Luo ZH, Spinney R, Wei ZS, Dionysiou DD, Zhao FP (2020a): An experimental and 

Environ 710

Xiao RY, Ma JY, Luo ZH, Zeng WZ, Wei ZS, Spinney R, Hu WP, Dionysiou DD (2020b): Experimental and theoretical insight into hydroxyl and sulfate radicals-mediated degradation of carbamazepine. Environ Pollut 257 trichlorophenol. Chemosphere 240 semiconductor catalyst to produce electric current or electron-holes pairs. Sci Rep 9, 5470 


\section{Figure captions}

439 Fig. 1 FTIR spectra of Fe-Mn hydroxide affected by the molar ratios of Fe(II) to Mn(II) (a) and the PS-

440 hydroxide influenced by the molar ratios of $\mathrm{Fe}(\mathrm{II})$ to $\mathrm{Mn}$ (II) (b), and PS-hydroxide influenced by the

441 mass ratios of persulfate to Fe(II)-Mn(II) hydroxide (c)

442 Fig. 2 Morphologies of Fe-Mn hydroxide affected by the molar ratios of Fe(II) to Mn(II) (a) and the PS-

443 hydroxide influenced by the mass ratios of PS to Fe-Mn hydroxide (b)

444 Fig. 3 Marginal means of the preparation parameters and aqueous conditions in the orthogonal design

$445 \quad$ (default $\alpha=0.05$ )

446 Fig. 4 Removal amounts of p-CP affected by the preparation parameters and aqueous conditions over $447 \quad$ time $(360 \mathrm{~min})$

448 Fig. 5 Linear fit of the kinetic model affected by the preparation parameters and aqueous conditions

Fig. 6 FTIR of p-CP before and after the degradation process (a) and the PS-hydroxide after the degradation process $(b)$

Fig. 7 Morphological characteristics of the PS-hydroxide after the degradation process

\section{Table legends}

Table 1 Orthogonal design with the removal amount of $\mathrm{p}-\mathrm{CP}$ for the preparation parameters

(Experimental conditions: $\mathrm{pH}$ of 7, the p-CP concentration of $6 \mathrm{mM}$, PS-hydroxide dosage of $1.5 \mathrm{~g} / \mathrm{L}$, and temperature of $50^{\circ} \mathrm{C}$ )

Table 2 Orthogonal design with the removal amount of p-CP for the aqueous conditions (Experimental conditions: the molar ratio of Fe(II) to Mn(II) of 3, the mass ratio of PS to Fe-Mn hydroxide of 3, the molar ratio of $\mathrm{OH}^{-}$to $\mathrm{Fe}(\mathrm{II})$ of 4) 

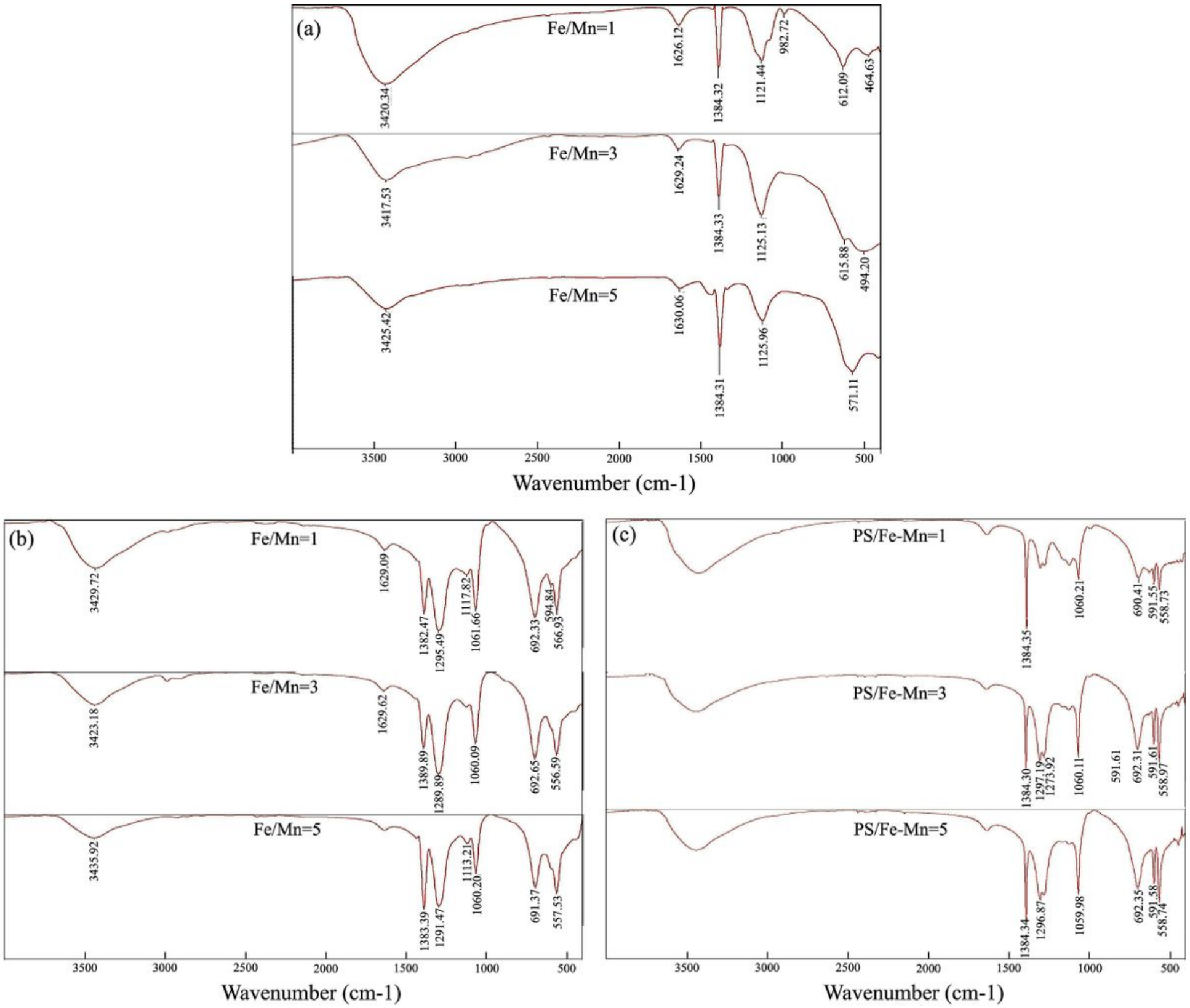

Figure 1

FTIR spectra of Fe-Mn hydroxide affected by the molar ratios of $\mathrm{Fe}(\mathrm{II})$ to $\mathrm{Mn}$ (II) (a) and the PS-hydroxide influenced by the molar ratios of $\mathrm{Fe}(\mathrm{II})$ to $\mathrm{Mn}$ (II) (b), and PS-hydroxide influenced by the mass ratios of persulfate to $\mathrm{Fe}(\mathrm{II})-\mathrm{Mn}$ (II) hydroxide (c) 

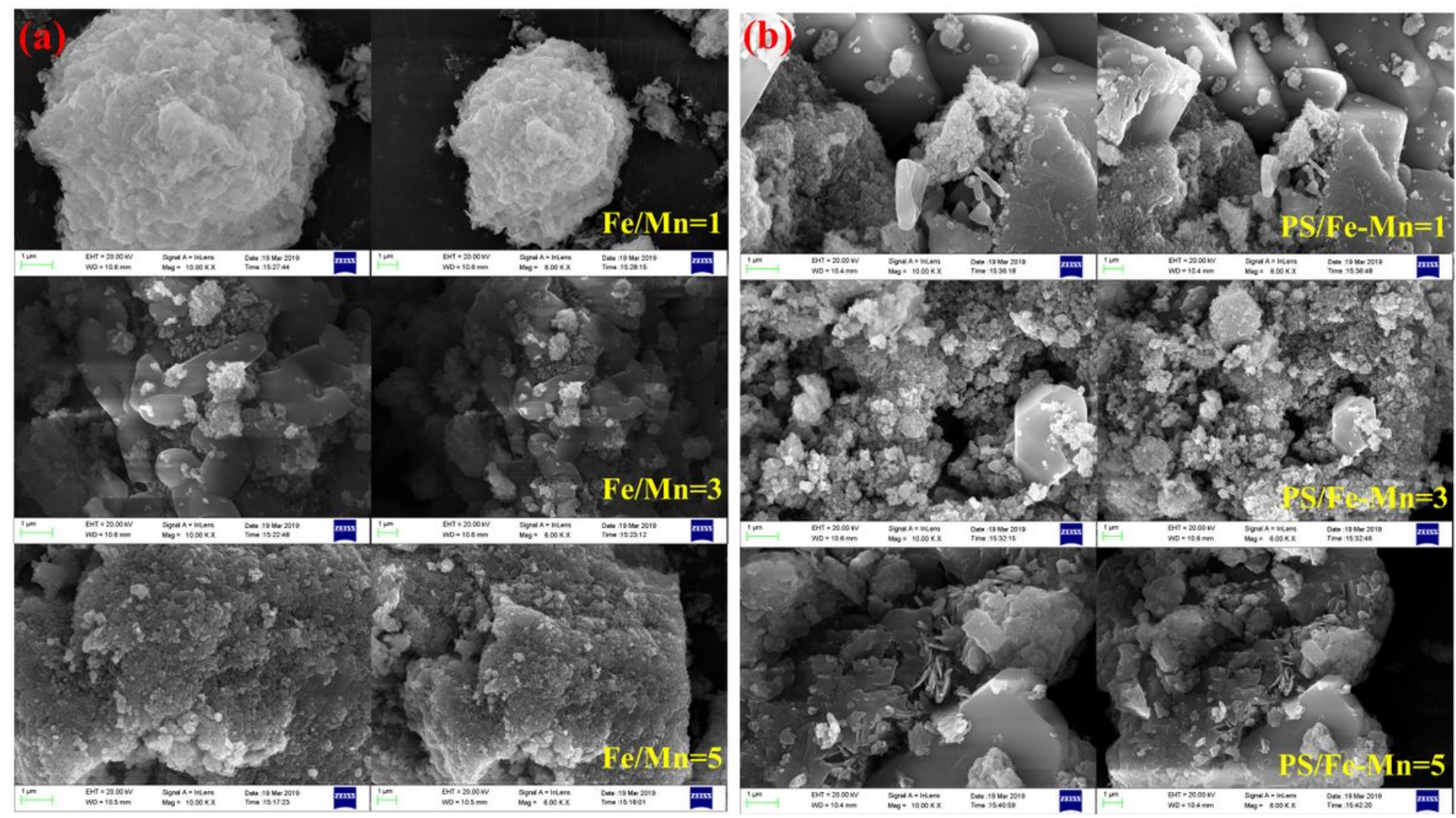

Figure 2

Morphologies of Fe-Mn hydroxide affected by the molar ratios of $\mathrm{Fe}(\mathrm{II})$ to $\mathrm{Mn}$ (II) (a) and the PS-hydroxide influenced by the mass ratios of PS to Fe-Mn hydroxide (b) 


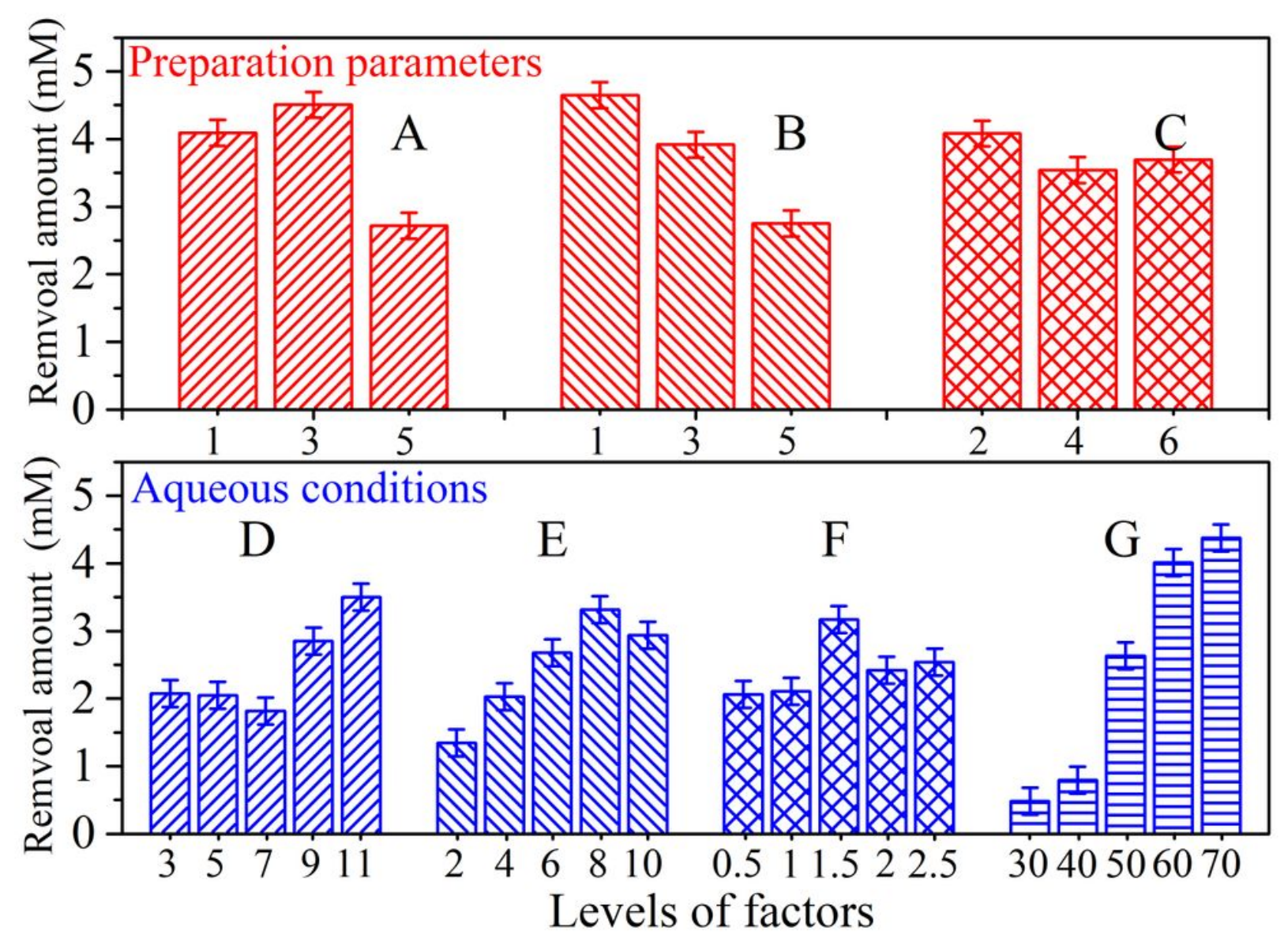

Figure 3

Marginal means of the preparation parameters and aqueous conditions in the orthogonal design (default $a=0.05)$ 

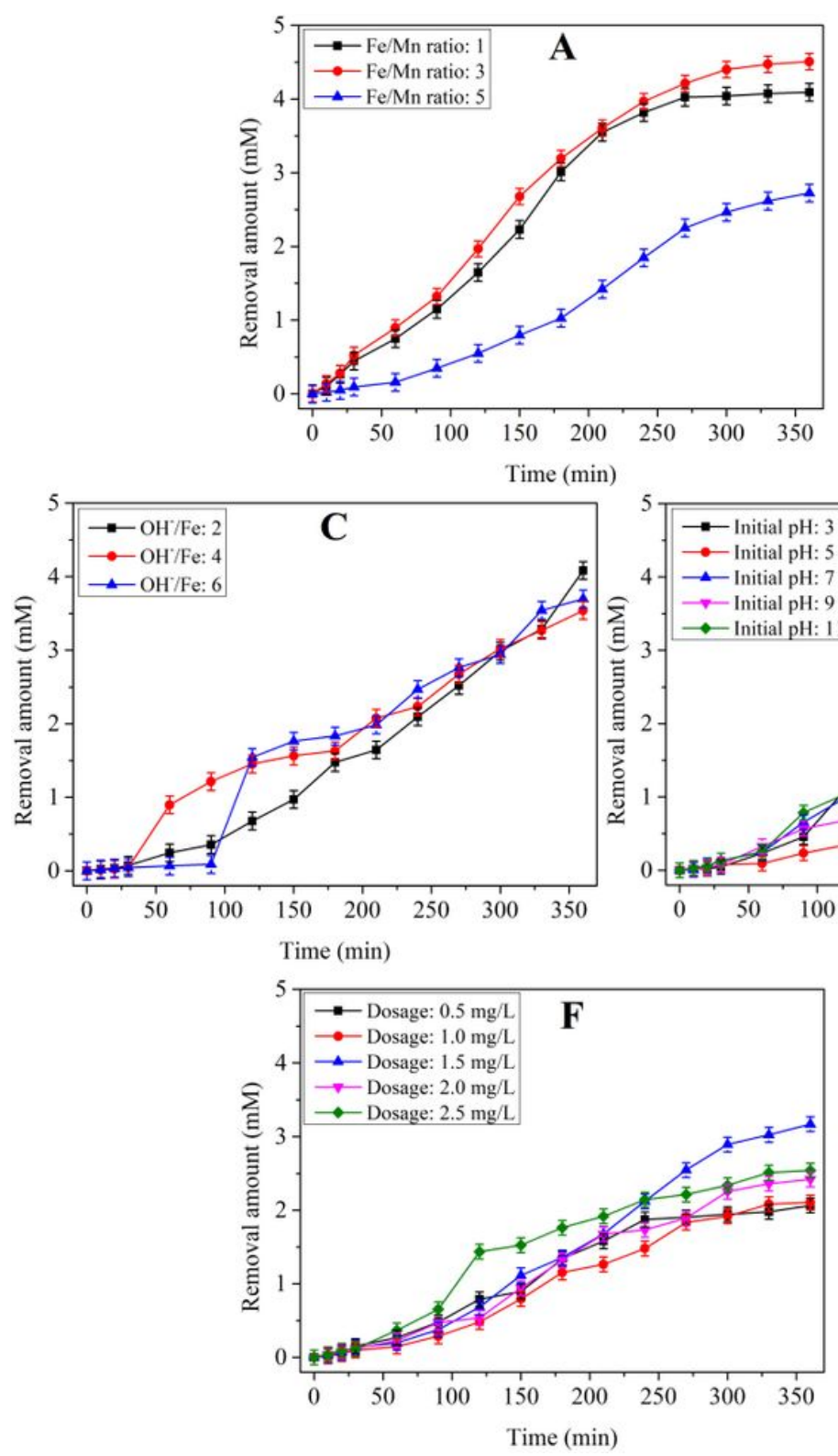
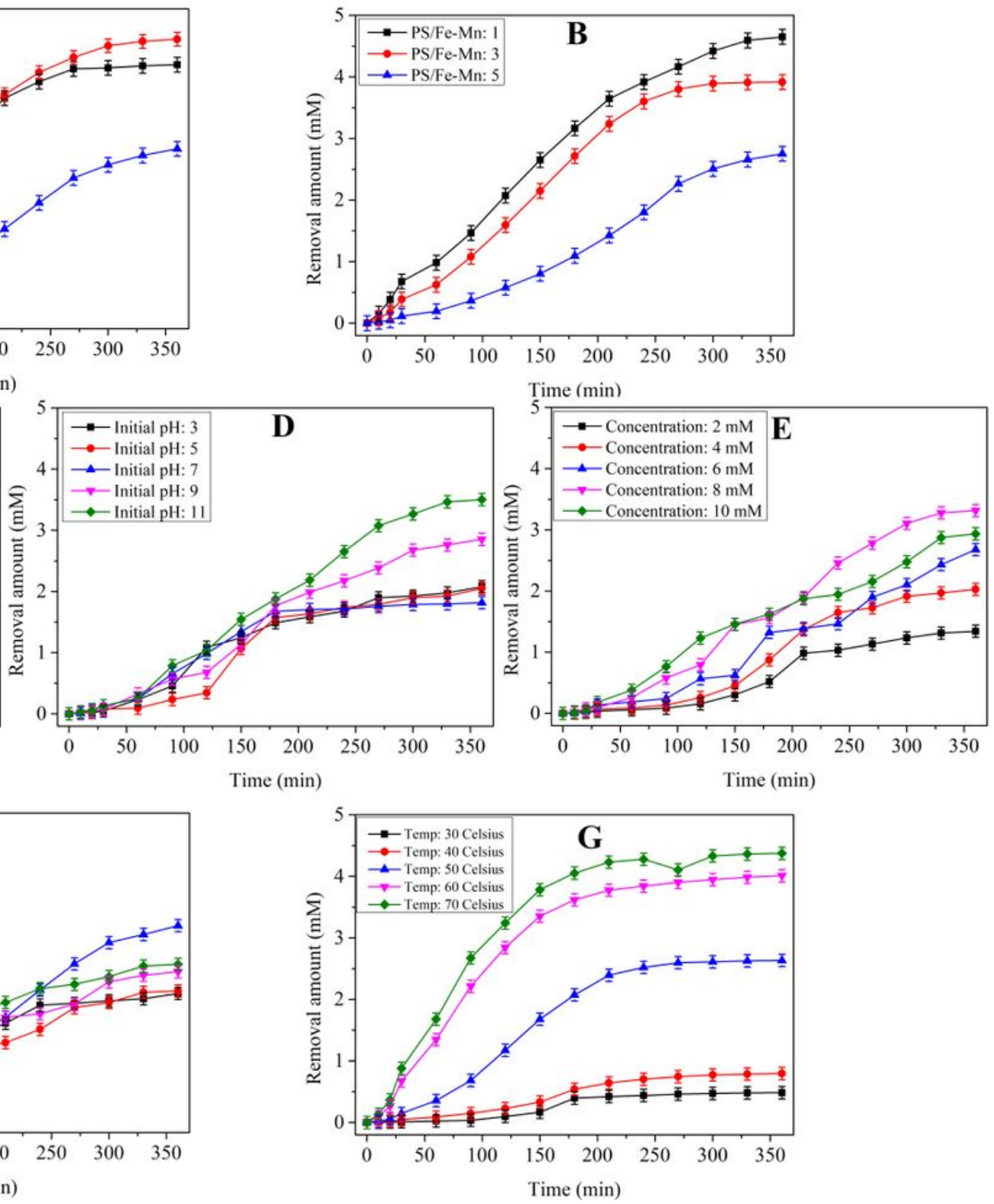

Figure 4

Removal amounts of $\mathrm{p}-\mathrm{CP}$ affected by the preparation parameters and aqueous conditions over time (360 min) 



Figure 5

Linear fit of the kinetic model affected by the preparation parameters and aqueous conditions 


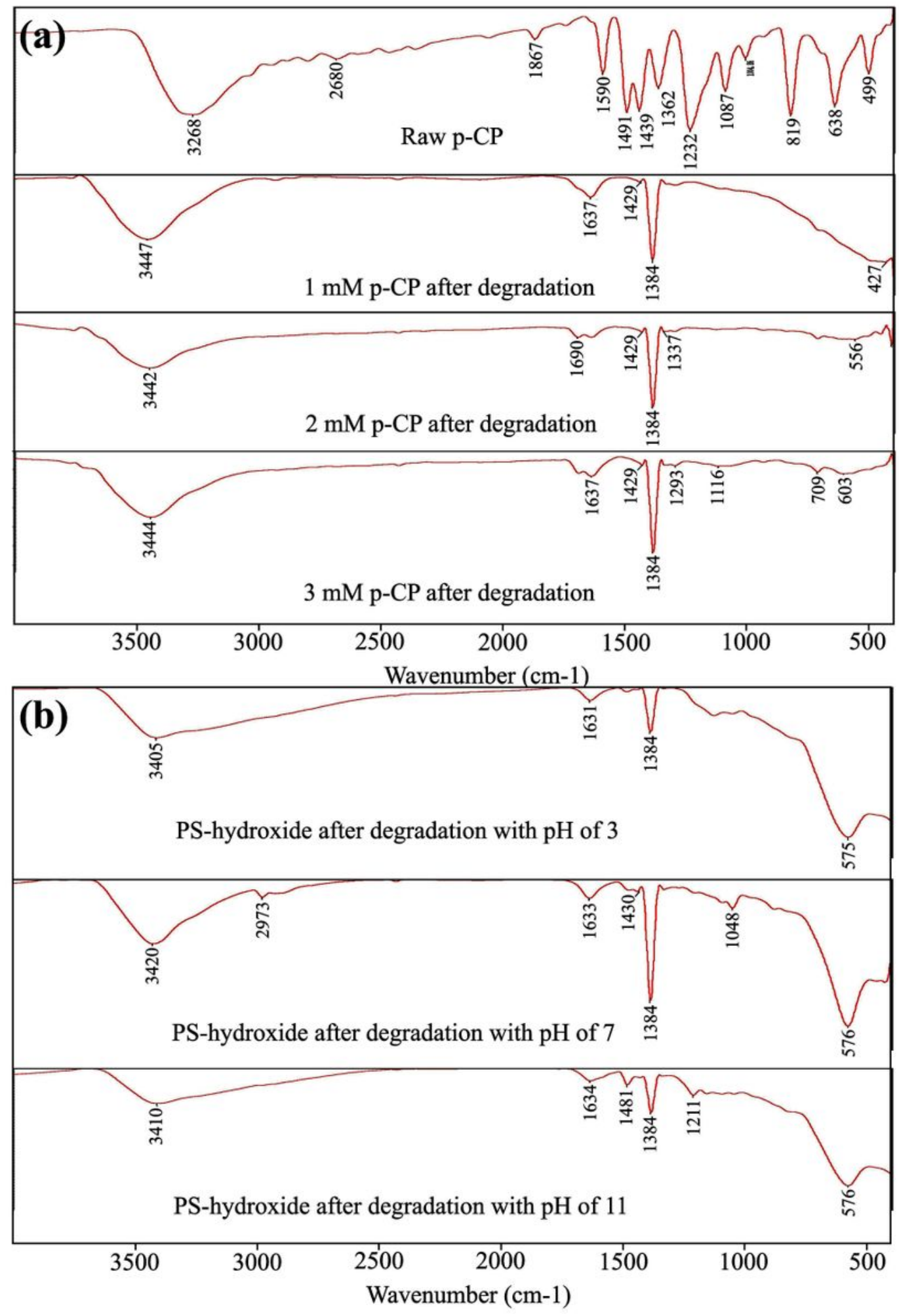

Figure 6

FTIR of $\mathrm{p}$-CP before and after the degradation process (a) and the PS-hydroxide after the degradation process (b) 


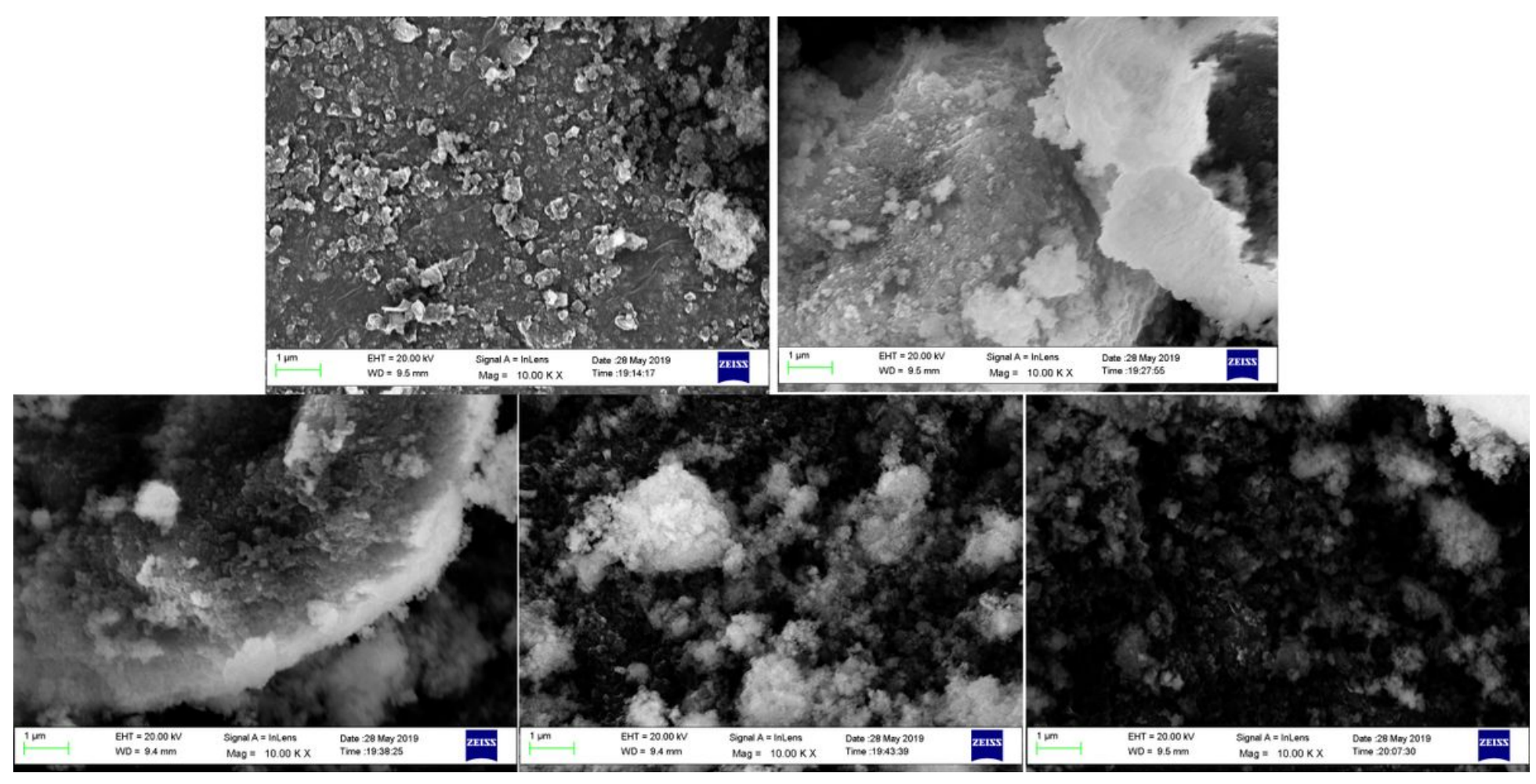

Figure 7

Morphological characteristics of the PS-hydroxide after the degradation process

\section{Supplementary Files}

This is a list of supplementary files associated with this preprint. Click to download.

- Supportinginformation.docx 